\title{
Probing long GRB progenitor mass by gravitational waves
}

\author{
Fabio Garufi* and Leopoldo Milano \\ Dipartimento di Fisica, Università di Napoli "Federico II" and INFN sez. di Napoli \\ Napoli, Italy \\ *E-mail: Fabio.Garufi@na.infn.it \\ www.unina.it \\ Mariafelicia De Laurentis \\ Tomsk State Pedagogical University, \\ Tomsk, 634061, Russian Federation \\ E-mail: mfdelaurentis@tspu.edu.ru
}

\section{Maria G. Dainotti}

Department of Physics \& Astronomy, Stanford University, Via Pueblo Mall 382 Stanford, CA 94305-4060 and Astronomical Observatory, Jagiellonian University, Krakow, Poland E-mail: maria.dainotti@riken.jp

\begin{abstract}
We present a procedure to infer the mass of progenitors and remnants of Gamma Ray Bursts (GRB), starting from the observed Electromagnetic energy emitted isotropically and considering the associated emission of Gravitatonal Waves (GW) in the different phases. Without making any assumption, we consider a purely empirical energy balance exclusively in GW energy with a GW emitting oblate progenitor, the energy emitted in GW during the GRB phase and the GW energy emitted by the residual object after the GRB (the remnant).

We take a sample of Long GRB, and use an hybrid Monte Carlo procedure to explore, for each of them, a region of possible solutions of GW energy as a function of the masses, radii, eccentricities, rotation frequencies of progenitor and remnant and the fraction of energy emitted as GW by the GRB.

We discriminate between a Neutron Star (NS) or Black Hole (BH) for the remnant and obtain interesting values for the GW emitted by the remnant NS or $\mathrm{BH}$, for the conversion factor and for the masses and radii of GRB progenitor stars. We also get remnant populations with mean masses, mean GW frequencies and GRB frequency of GW emission in agreement with the most accepted models.
\end{abstract}

Keywords: Gravitational waves; gamma ray bursts; stellar collapse.

\section{Introduction}

We consider here the fraction of LGRBs coming from a collapse resulting in a SN explosion, making the euristic assumption that the energy radiated in GW is a fraction of the total emitted energy.

In cases where the GWs emission comes from a core-collapse process, we cannot predict the exact shape of the emitted GWs signal, but we can have an estimate starting from the energy flux ${ }^{1}$ :

$$
\mathcal{F}_{\mathrm{GW}}=\frac{d^{2} E^{\mathrm{GW}}}{d t d S}=\frac{c^{3}}{16 \pi G}\left\langle\left(\dot{h}_{+}\right)^{2}+\left(\dot{h}_{\times}\right)^{2}\right\rangle,
$$

where $\langle\ldots\rangle$ indicates a temporal average over a large enough number of periods. The 
total energy emitted assuming isotropic emission is then

$$
E^{\mathrm{GW}}=4 \pi D^{2} \int d t \mathcal{F}_{\mathrm{GW}}
$$

where $D$ is the distance to the source. Thus, in terms of frequencies:

$$
P^{\mathrm{GW}}=\frac{\pi^{2} c^{3}}{4 G} D^{2} \int_{-\infty}^{\infty} f \tilde{h}_{0}(f) e^{-2 \pi i f t} d f \int_{-\infty}^{\infty} f^{\prime} \tilde{h}_{0}^{*}\left(f^{\prime}\right) e^{2 \pi i f^{\prime} t} d f^{\prime},
$$

being $\tilde{h}_{0}^{2}(f)=\sqrt{\left(\tilde{h}_{+}\right)^{2}+\left(\tilde{h}_{\times}\right)^{2}}$. If the frequency distribution of the GRB is peaked around a frequency $f_{G W}$, as e.g. in a sine-Gaussian waveform, eq. (3) can be written as:

$$
P^{\mathrm{GW}}=\frac{\pi^{2} c^{3}}{4 G} D^{2} 2 f_{G W}^{2} h_{0}(t)^{2} .
$$

Without making any hypothesis on the mechanism generating GWs in the SN explosion, here we simply assume that the energy emitted in gravitational waves is a fraction $k$ of $E_{\text {iso }}^{G R B}$, we have:

$$
P^{\mathrm{GW}}=\frac{k E_{i s o}^{G R B}}{T_{90}}
$$

where $T_{90}$ is the time in which the $90 \%$ of $E_{i s o}^{G R B}$ is emitted, and so:

$$
h_{0_{\mathrm{GW}}}^{2}(t)=\frac{k E_{i s o}^{G R B}}{T_{90} \pi^{2} D^{2} f_{G W}^{2}} \frac{G}{c^{3}} .
$$

In the following we will call "remnant" what remains after GRB phase, being it a NS or a BH. When necessary we will specify the type of remnant we are dealing with.

Let us consider a progenitor star of mass $M_{P R G}$, radius $R_{P R G}$ and oblateness $\epsilon_{P R O G}$, rotating with a frequency $f_{P R G}=0.5 f_{P R G_{G W}}$, and a NS as a remnant after the process of GRB emission with parameters $M_{N S}, R_{N S}, \epsilon_{N S}$ and $f_{N S}$. We have that $^{1}$ :

$$
\begin{aligned}
h_{\mathrm{GW}}^{P R G} & =\frac{4 \pi^{2} G}{c^{4}}\left(\frac{I_{3}^{P R G} f_{P R G_{G W}}^{2}}{D} \epsilon_{P R O G}\right), \\
h_{\mathrm{GW}}^{N S} & =\frac{4 \pi^{2} G}{c^{4}}\left(\frac{I_{3}^{N S} f_{G W_{N S}}^{2}}{D} \epsilon_{N S}\right) .
\end{aligned}
$$

The momentum of inertia for a spherical rigid object (considering negligible the quadrupolar oblateness $\epsilon$ ) is given by:

$$
I_{3}^{N S}=\frac{2}{5} M_{N S} R_{N S}^{2} ; \quad I_{3}^{P R G}=\frac{2}{5} M_{P R G} R_{P R G}^{2}
$$

We can roughly say that the energy emitted in GW by the GRB is given by the difference of the energies emitted in GRB by the progenitor and the remnant, resulting in a similar relationship between the squares of GW amplitudes. From eqs. 
(6), (7), (8), (9), we can very roughly write, in terms of the only observables, i.e. $E_{i s o}^{G R B}$ and T90:

$$
E_{\text {iso }}^{G R B}=\frac{64 G \pi^{6} T_{90} f_{G W}^{2}\left(M_{P R G}^{2} R_{P R G}^{4} f_{P R G}^{4} \epsilon_{P R G}^{2}-M_{N S}^{2} R_{N S}^{4} f_{N S}^{4} \epsilon_{N S}^{2}\right)}{25 c^{5} k},
$$

\section{Genetic controlled random search algorithm for GRB progenitor masses estimate}

Our aim is to find the best progenitor mass and the type of remnant star, starting from the observed $E_{i s o}^{G R B}$. Given (Eq. (10) we see that, from the observed $E_{i s o}^{G R B}$ we must infer ten parameters. To solve the problem we use an algorithm that is capable to find the global minimum of a multivariable function. We used a simplified simplex algorithm: the controlled random search (CRS) algorithm and, to improve its performances, we used a genetic modification of the search procedure making the software more resilient to local minima ${ }^{2-5}$.

Since we have 10 parameters to estimate from only 2 observables, for each observed GRB, we generate a large number $N_{G R B}$ of $E_{i s o}$ values extracted randomly from a Gaussian distribution with mean value $E_{\text {isoobs }}$ and standard deviation $\sigma_{E_{i s o}}$ given by the observed measurement error; then we build the objective function to minimize, using the reduced $\chi_{o b s}^{2}$ with $N_{G R B}-N$ degrees of freedom, where $N=10$ is the number of free parameters:

$$
F_{O b}=\chi_{o b s}^{2}=\frac{1}{N_{G R B}-N} \sum_{i=1}^{N_{G R B}} \frac{\left(E_{\text {iso }_{i} \text { obs }_{\text {o }}}-E_{\text {iso }_{i_{C}}}\right)^{2}}{\sigma_{\text {iso }}^{2}},
$$

where $E_{i s o_{[O b s, C]}}$ are the observed and computed $E_{i s o}$ obtained from Eq. (10) as a function of the parameters to find. Finally we minimize $\chi_{O b s}^{2}$ with $N_{G R B}-N$ degrees of freedom up to $5 \%$ of confidence level $\left(\chi_{O b s}^{2} \leq 0.86\right)$.

\section{Results}

We selected a sample of 237 long GRBs, detected by the Swift satellite ${ }^{6}$ from January 2005 to May 2014 with known redshifts, that we then analyzed. This sample is an extended sample as from Refs. 7,8 where GRBs with plateaus are presented. In Table 1 the sample GRB data range, that we analyzed, is shown. Considering the

Table 1. Sample GRB data range used in this work.

\begin{tabular}{|l|l|l|l|l|l|}
\hline$E_{\text {iso }}^{G R B}(\mathrm{erg})$ & $\sigma_{E_{\text {iso }}^{G R}(\mathrm{erg})}$ & $T 90(\mathrm{~s})$ & $\sigma_{T 90}(\mathrm{~s})$ & redshift $z$ & $D \times 10^{26}(\mathrm{~cm})$ \\
\hline $2.5 \times 10^{48} \div 1.1 \times 10^{55}$ & $3.9 \times 10^{47} \div 1.7 \times 10^{54}$ & $2.2 \div 844$ & $0.003 \div 3$ & $0.014 \div 8.2$ & $1.8 \div 2500$ \\
\hline
\end{tabular}

above mentioned sample of long GRBs and according to the hypotheses we made, it is possible to infer the physical parameters of the GRB progenitor with their errors. We allow the parameters to vary in 4 possible regions of solutions according to the 
Table 2. Mean results for model parameters obtained from genetic-Price algorithm.

\begin{tabular}{|l|l|l|l|l|l|}
\hline Model & $M_{P R O G}\left(M_{\odot}\right)$ & $R_{P R O G}\left(R_{\odot}\right)$ & $M_{\operatorname{Remn}}\left(M_{\odot}\right)$ & $R_{\operatorname{Remn}}(\mathrm{km})$ & $f_{\operatorname{Remn}}(\mathrm{Hz})$ \\
\hline Model 1 & $41 \pm 5$ & $50 \pm 12$ & $2.3 \pm 0.7$ & $10 \pm 2$ & $504 \pm 260$ \\
\hline Model 2 & $34 \pm 3$ & $36 \pm 11$ & $5 \pm 2$ & $16 \pm 6$ & $782 \pm 300$ \\
\hline Model 3 & $25 \pm 4$ & $11 \pm 4$ & $2.0 \pm 0.5$ & $9 \pm 3$ & $495 \pm 200$ \\
\hline Model 4 & $25 \pm 4$ & $10 \pm 4$ & $5 \pm 2$ & $15 \pm 6$ & $493 \pm 230$ \\
\hline
\end{tabular}

Table 3. Parameters common to the different model of Table 2 useful to initialize the domain of possible solutions.

\begin{tabular}{|l|l|l|l|l|}
\hline$k$ & $f_{G R B}^{G W}(\mathrm{~Hz})$ & $\omega_{P R O G}(\mathrm{rad} / \mathrm{s})$ & $\epsilon_{P R O G}$ & $\epsilon_{R e m n}$ \\
\hline $10^{-9} \div 10^{-4}$ & $50 \div 800$ & $10^{-4} \div 10^{-10}$ & $10^{-2} \div \frac{2}{3}$ & $10^{-8} \div 10^{-4}$ \\
\hline
\end{tabular}

grids of stellar models, that we call models 1-4: The mean results of parameters common to the different models of Table 2 are shown in Table 3.

By comparing the emission of GW from the remnants with the sensitivity curves of the GW detectors: LIGO, VIRGO Advanced Ligo AdvVirgo (Fig. 3), where an interesting prediction of a remnant population of either millisecond pulsar (Model 1-Model 3) or BH (Model 2-Model 4) can be noticed, the non detection so far of any signal, rules out all models except Model $3^{9}$. We show here the also result for two well studied GRBs:

The case of a $\mathrm{BH}$ remnant, of course, is not well modeled by our treatment, as one should expect a much larger GW emission, nonetheless, also considering our result as a lower limit, the non observation yet excludes the $\mathrm{BH}$ outcome.

Table 4. Progenitor mass in $M_{\odot}$, progenitor radius $R_{P R O G}$ in $R_{\odot}$. Remnant masses, radii and GW remnant emission frequency of GRB030329 and GRB060218 according to Model 3.

\begin{tabular}{|l|l|l|l|l|l|}
\hline & $M_{P R O G}$ & $R_{P R O G}$ & $M_{\text {Remn }}$ & $R_{\operatorname{Remn}}(\mathrm{km})$ & $f_{\text {Remn }}(\mathrm{Hz})$ \\
\hline GRB 030329 & $24 \pm 10 M_{\odot}$ & $10 \pm 5 R_{\odot}$ & $2.0 \pm 0.8 M_{\odot}$ & $10 \pm 4$ & $491 \pm 280$ \\
\hline GRB 060218 & $21 \pm 8 M_{\odot}$ & $8 \pm 5 R_{\odot}$ & $2.0 \pm 0.5 M_{\odot}$ & $8 \pm 3$ & $393 \pm 250$ \\
\hline
\end{tabular}

For the case of GRB060218 it is easy to see that both the progenitor masses and radii computed according to model 3 , are in good agreement with the estimations by Campana et $\mathrm{l}^{12}$ stating that observations provide strong evidence that the GRB progenitor was a WR star, being the star radius definitely smaller than $5 \times 10^{12} \mathrm{~cm}$, i.e. smaller than the radius of the progenitors of type II SNe, like blue supergiants $\left(4 \times 10^{12} \mathrm{~cm}\right.$ for SN1987A) or red supergiants $\left(3 \times 10^{13} \mathrm{~cm}\right)$. 


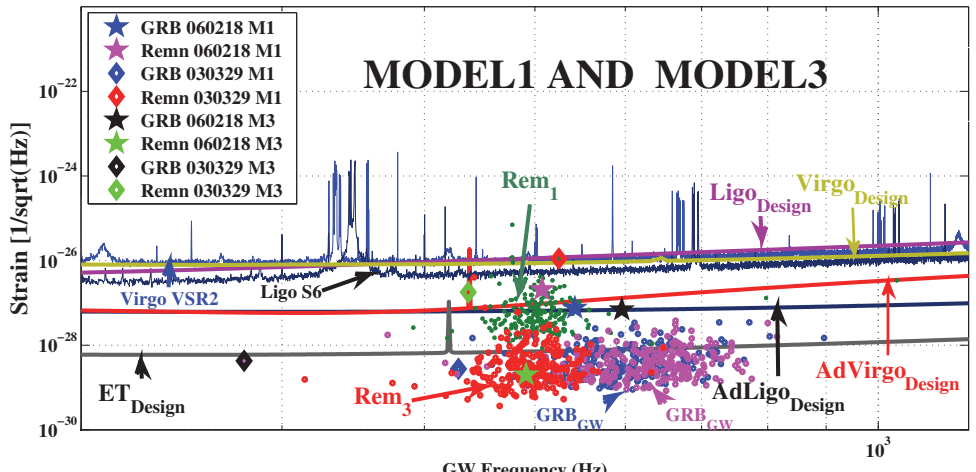

GW Frequency (Hz)

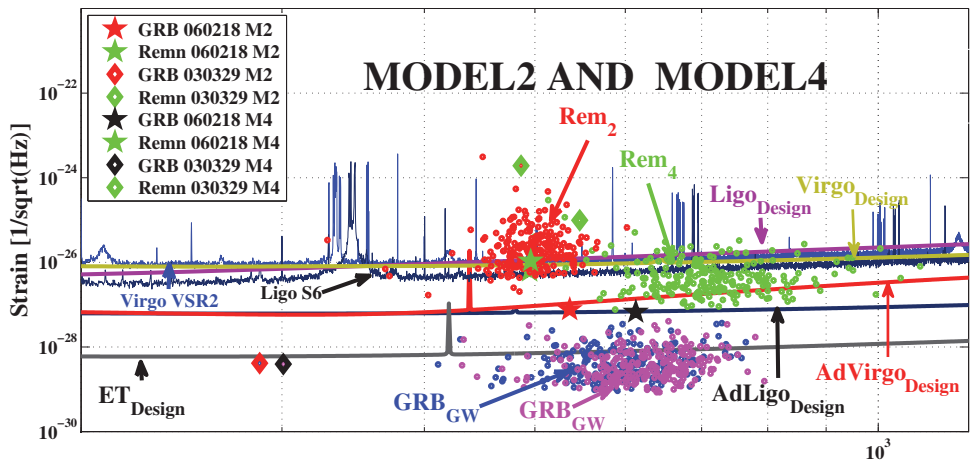

GW Frequency $(\mathrm{Hz})$

Fig. 1. On top panel GW amplitudes for the GRB sample are shown for models 1 and 3 (blue and magenta circles respectively) together with GW emission by remnants (NS for these models, red and green circles) and overlapped to VIRGO and LIGO sensitivity curves from the scientific run (VIRGO) VSR2 and the scientific S6 run (LIGO), compared with the respective target curves ${ }^{10,11}$. The GW amplitudes of GRB060218 and GRB030329 are also shown. On bottom panel the same is shown for models 2 and 4 (BH remnants). An interesting prediction of a remnant population of either millisecond pulsar (Model 1-Model 3: green and red circles top) or BH (Model 2-Model 4: red and green circles bottom) can be noticed. 


\section{References}

1. Maggiore M., 2007, Gravitational Waves: Theory and Experiments, Oxford Univ. Press, Oxford.

2. Price W.L. 1976, Computer J., 7,303

3. Milano L., Barone F.,Milano M.,1997, Phys. Rev. D, 55,6 .

4. Milano M., Koumoutsakos P., 2002 Journ of Comput. Physics, 175, 79-107.

5. Bresco M. et. al., 2005, Bresco M., SOFT COMPUTING 9, pp 525-535.

6. Gehrels, N., et al. 2004, ApJ,611, 1005.

7. Dainotti M.G., Petrosian V., Singal J., Ostrowski M., 2013, Astrophys.J. 774, 157.

8. Dainotti M.G., Cardone V.F., Piedipalumbo E., Capozziello S., 2013, Mon. Not. Roy. Astron. Soc. 436, 82.

9. M. Delaurentis, F. Garufi, M.G.Dainotti, L. Milano, arXiv:1506.00106 (2015)

10. Abadie J. et al., 2012, ApJ 760, 12.

11. Abbott B.P. et al., 2010, ApJ 715, 1438.

12. Campana S. et al., 2006, Nature 442, 1008. 